\title{
Polarimetric Characterization of Debris and Faults in the Highway Environment at Millimeter-Wave Frequencies
}

\author{
Kamal Sarabandi, Fellow, IEEE, and Eric S. Li
}

\begin{abstract}
In this paper, measurements and models for the polarimetric backscatter response of various point targets on roads and road surface faults is presented. Of particular interest are debris and faults that could lead to fatal accidents and damage of property. A desired safety feature for automotive radar sensors is the capability of detecting such debris and faults. The detectability of a point target is evaluated by comparing its RCS value with the RCS threshold value defined by the backscatter response of the road surface. Extensive backscatter measurements at W-band were conducted to obtain the backscatter response of typical debris and faults on asphalt surfaces at near grazing incidence angles $\left(76^{\circ}-86^{\circ}\right)$. On the other hand, theoretical models, based on diffraction from impedance wedges and scattering from impedance cylinders, respectively, as well as physical optics approximation, were developed to predict the backscatter response of road surface faults and targets with planar facets on road surfaces. Experimental results indicate that detectability in all cases is a function of target size, its azimuthal angle with respect to radar boresight, and the polarization state of the system. The measured backscatter response is used to verify the validity of the theoretical models. Angular polarimetric backscatter measurements of targets defining roadside boundaries such as a concrete curb, a guardrail, and a pebble surface are also presented. The results of these measurements could be used to alert fatigued drivers should their vehicles be heading sideward.
\end{abstract}

\section{INTRODUCTION}

$\mathbf{E}$ $\mathrm{ACH}$ year, numerous automotive accidents are reported. Many of these are attributed to road-surface conditions and objects or obstacles on road surfaces. Design of a reliable automotive radar sensor requires thorough knowledge of backscatter response of road surfaces and typical debris and faults on road surfaces. The problem of polarimetric radar scattering from road surfaces are considered in two earlier papers [1], [2] where accurate theoretical models are developed for the determination of backscatter response of asphalt and concrete surfaces under wide varieties of physical conditions. These models are successfully compared with careful backscatter measurements of bare and water-, ice-, and snow-covered smooth and rough asphalt and concrete surfaces over a wide range of incidence angles at $\mathrm{W}$-band frequencies. A potential application of these models and measurements are demonstrated in [3] where the design of an affordable millimeter-wave (MMW) radar is proposed for determination of road-surface conditions. A desirable feature of

Manuscript received October 28, 1998; revised July 17, 2000.

The authors are with the Radiation Laboratory, Department of Electrical Engineering and Computer Science, The University of Michigan, Ann Arbor, MI 48109 USA (e-mail: saraband@eecs.umich.edu).

Publisher Item Identifier S 0018-926X(00)10835-X automotive radar sensors is the detection of debris and faults in the highway environment and the provision of an advance warning to drivers. The debris commonly encountered on the highway with potential threat to vehicle safety are bricks, scrap metal, bolts, etc. Potholes and cracks are typical faults seen on road surfaces. Debris and faults may be detected by comparing the radar cross-section (RCS) values of the targets on asphalt surfaces with the backscatter from the asphalt surfaces alone. The backscatter from road surfaces is statistical in nature and as a result has a wide fluctuation range. Therefore, in order to detect an obstacle with a very low false-alarm rate, the RCS of the target should be larger than the maximum RCS of the surfaces for a prescribed confidence range. The backscatter response of some nonhazardous targets such as cracks on road surfaces, which generate strong backscatter should also be determined and compared with the RCSs of desired targets for the evaluation of false-alarm rate.

Since the RCS of a point target is influenced by many physical parameters such as material, size, shape, and target orientation, a complete polarimetric characterization of debris and faults on road surfaces would be very difficult. This problem can be solved by resorting to analytical solutions. Two theoretical models are developed to predict the backscatter response of cracks perpendicular to the antenna boresight. The first model simulates a crack by two impedance wedges next to each other. The method developed by Maliuzhinets [4], [5] is used to calculate the total field in the presence of an impedance wedge at normal incidence condition. A nonuniform expression for impedance wedge diffraction is chosen to represent the diffraction coefficient. The diffracted fields from two adjacent wedges are then added coherently. At high MMW frequencies, the radius of curvature of asphalt or concrete crack edges become comparable with the wavelength and the wedge model may become invalid. For this situation, a second model for curved edges based on the scattering from impedance cylinders is developed. The simulation results of the second model exhibit better agreement with the measured data at $94 \mathrm{GHz}$ than do those of the first model. Also, a physical-optics model is developed for predicting scattering from solid objects with perpendicular facets such as bricks and right-angled iron, and, simulation results are compared with the backscatter measurements of a brick and a piece of right-angled iron on an asphalt surface at $94 \mathrm{GHz}$.

This paper begins with a description of the experimental setup for the backscatter measurements of debris and faults on asphalt surfaces. Then, the RCS threshold value is defined in terms of the antenna gain function, the antenna height, and the 
backscattering coefficients of the road surfaces. This value is used to determine the detectability of various debris and faults on asphalt surfaces. Next, the backscatter response of debris on asphalt surfaces is examined. The polarimetric backscatter measurements of lane-guide reflectors are also included. The detectability of the debris and reflectors is then evaluated. The backscatter behavior of faults on asphalt surfaces is described in Section IV along with investigation of their detectability. The backscatter characterization of typical roadside boundaries such as pebbles, curbs, and guardrails is also performed experimentally. Detection of these targets is important because of the fact that drowsiness and fatigue are among the leading causes of sideward crashes. Besides alerting the drowsy driver, the backscatter response could also provide valuable information for adaptive cruise-control applications. Section V includes the backscatter response of the roadside boundaries.

\section{Measurement SetuP And Detectability}

The University of Michigan 94-GHz fully polarimetric radar system was employed to conduct the backscatter measurements. The system bandwidth is set to $1 \mathrm{GHz}$ to improve the range resolution of the point target response for time-domain gating during postprocessing. Finite asphalt surfaces of dimension $91.5 \mathrm{~cm}$ $\times 91.5 \mathrm{~cm} \times 6.4 \mathrm{~cm}$ were constructed for the indoor measurements. These sample surfaces could easily be mounted on a turnable for RCS pattern measurements of point targets on asphalt surfaces. The backscatter response of most point targets on an asphalt sample surface was measured at incidence angles from $76^{\circ}$ to $86^{\circ}$. The quantities of interest are the backscatter from the point targets and their interactions with the asphalt surface. The leading edges of asphalt samples were covered by radar-absorbing material to reduce edge diffraction at near grazing incidence. Background subtraction and range gating were used to remove the unwanted signals, followed by a calibration procedure to remove systematic errors such as channel imbalance and antenna crosstalk from the measured data [6].

As aforementioned, road surfaces at MMW frequencies can be modeled as random media whose backscatter is statistical in nature and is usually composed of surface and volume scattering components [1], [2]. Since in most practical situations the radar footprint covers a large number of scatterers over the asphalt surface and within the asphalt medium, according to the central limit theorem, the backscattered field obey a Gaussian statistics. It is well known that statistics of the backscatter field intensity (power) has exponential distribution given by

$$
f_{\sigma}\left(\sigma_{a}\right)=\frac{1}{\bar{\sigma}} e^{-\left(\sigma_{a} / \bar{\sigma}\right)}
$$

where $\bar{\sigma}$ is the mean backscatter from the illuminated portion of the distributed target (asphalt surface). This mean value is a function of the target backscatter coefficient $\sigma^{\circ}$, the antenna pattern, incidence angle, and antenna height

$$
\bar{\sigma}=\sigma^{\circ} \iint_{\text {LitArea }} \frac{G^{2}(x, y)}{r^{4}(x, y)} d x d y=\sigma^{\circ} A_{\text {illu }}
$$

where $G(x, y)$ and $r(x, y)$ are the antenna gain and the distance from the antenna to a point within the lit area, respectively, and

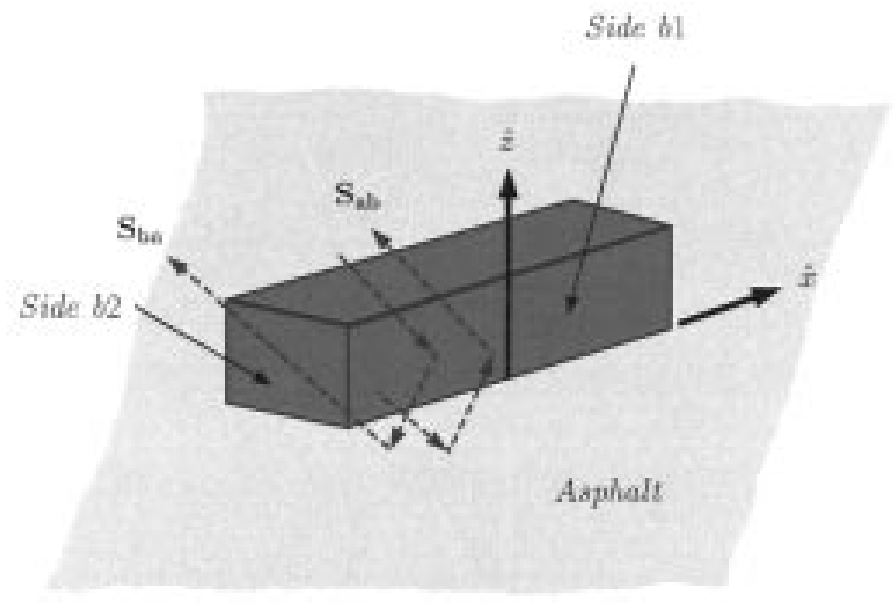

Fig. 1. The scattering mechanisms of the backscatter response of the brick on asphalt surfaces.

$A_{\text {illu }}$ is known as effective illuminated area. To examine the detectability of a point target over an asphalt surface with a reasonable false-alarm rate (FAR), the RCS of the illuminated area of asphalt $\left(\sigma_{a}\right)$ must be compared with the RCSs of the point targets $\left(\sigma_{p}\right)$. Choose a threshold level $\sigma_{a}^{\text {th }}$ based on a given maximum false-alarm rate, a criteria for target detectability is obtained. For a maximum false-alarm rate (MFAR), $\sigma_{a}^{\text {th }}$ is set so that

$$
\int_{0}^{\sigma_{a}^{\text {th }}} f_{\sigma}\left(\sigma_{a}\right) d \sigma_{a}=1-\text { MFAR. }
$$

Using (1) in (3) we find

$$
\sigma_{a}^{\text {th }}=-\bar{\sigma} \ln (\mathrm{MFAR})
$$

Note that the RCS quantities in (4) are in linear scale. Hence, for a MFAR of $10^{-5}$, the RCS of the point targets must be $10.6 \mathrm{~dB}$ above $\bar{\sigma}=\sigma^{\circ} A_{\text {illu. }}$. It is obvious that smaller targets can be detected reliably for antenna with small footprint. This threshold value $\left(\sigma_{a}^{\text {th }}\right)$ used in this investigation is obtained from the backscattering coefficients $\left(\sigma^{\circ}\left(\theta_{i}\right)\right)$ of a smooth asphalt surface measured by a single-antenna radar mounted at vehicle bumper height $(43 \mathrm{~cm})$ and the gain function of the antenna is approximated by a Gaussian function:

$$
G(\theta, \phi)=e^{-a_{\odot} \theta^{2} / \beta^{2}}
$$

where $a_{\circ}$ is 2.7726 and $\beta$ is the half-power beamwidth, equal to $1.4^{\circ}$. The detectability of point targets on asphalt surfaces is examined at an incidence angle of $80^{\circ}$.

\section{Debris AND LANE-GUide REFLECTORS ON ROAD SURFACES}

In this section, the backscatter measurements and models for some points targets on asphalt surfaces are presented and their detectability is examined. Three common types of highway debris are considered: bricks, scrap metal, and bolts. Also, the measured backscatter behavior of lane-guide reflectors as a function of aspect angle is presented here. 


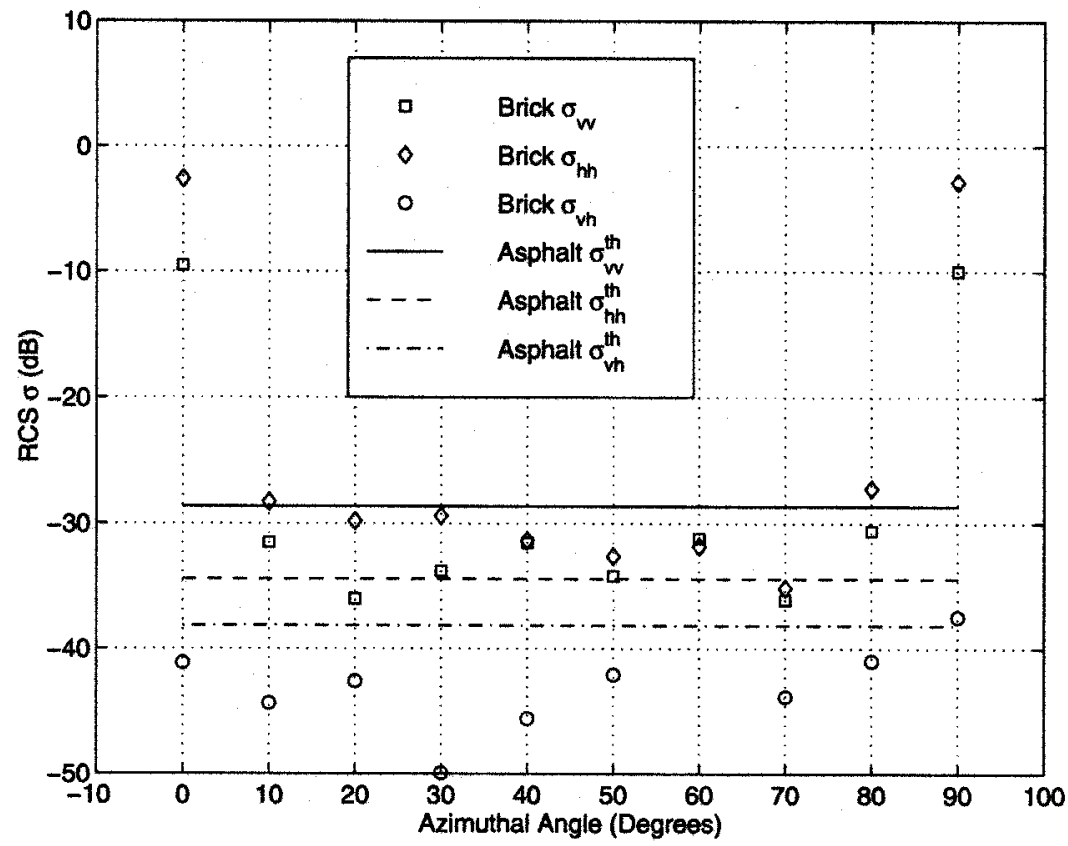

Fig. 2. The backscatter response of the brick on asphalt surfaces at an incidence angle of $80^{\circ}$.
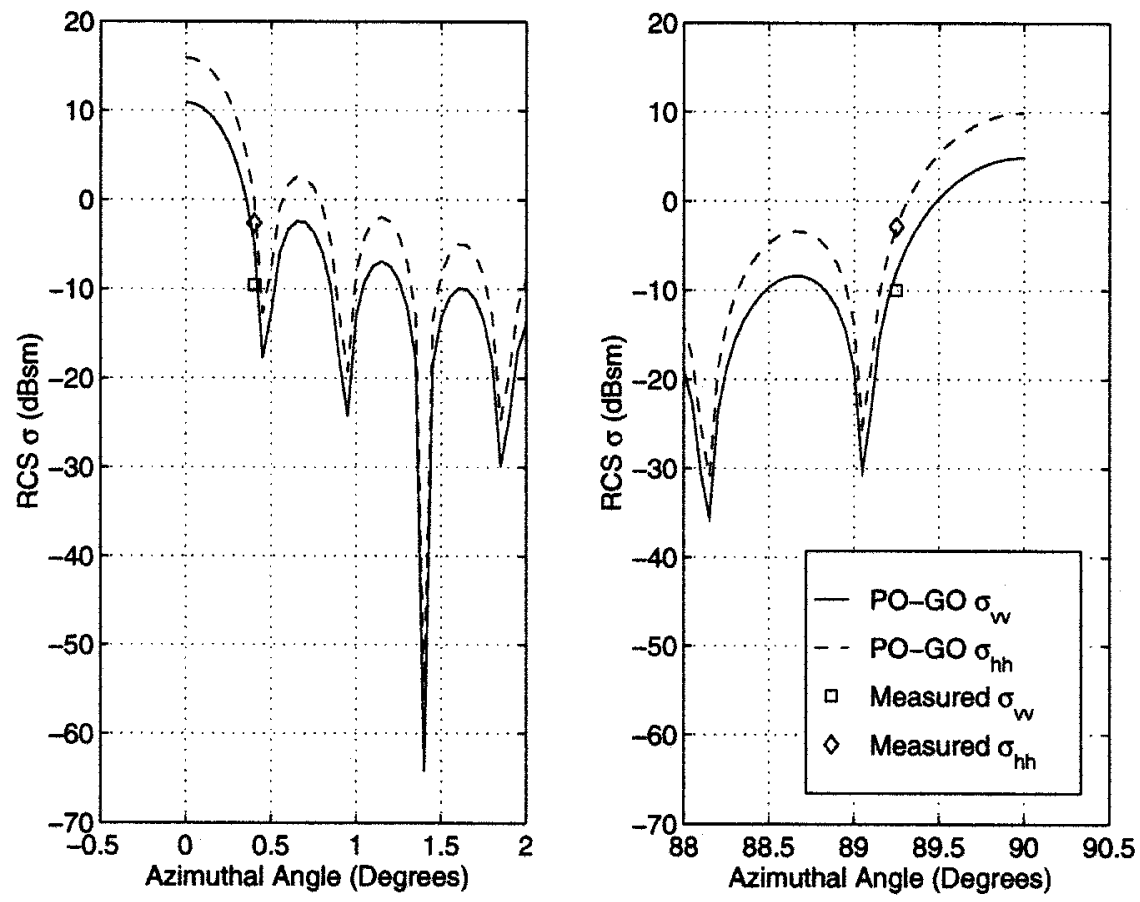

Fig. 3. At an incidence angle of $80^{\circ}$, the theoretical values of the backscatter RCSs of the brick on asphalt surfaces are compared with the measured data at azimuthal angles near $0^{\circ}$ and $90^{\circ}$.

\section{A. Targets with Planar Facets}

A simple scattering model based on physical optics (P. O.) approximation for targets with planar surfaces, such as bricks and right-angled iron, is developed and the results are compared with backscatter measurements. At W-band frequencies the sizes of most targets of interest (those that create hazardous conditions for automobiles) are large compared to the wavelength and their relative permittivity has a sufficiently large imaginary part, thus PO model is expected to provide accurate results.

The backscatter response of a brick on an asphalt surface can be obtained from that of a planar dielectric surface above an infinite ground plane (asphalt surface). The bistatic scattering from a planar dielectric surface whose dimensions are large compared to the wavelength can be modeled using the PO approximation. The expression for the bistatic far-field 


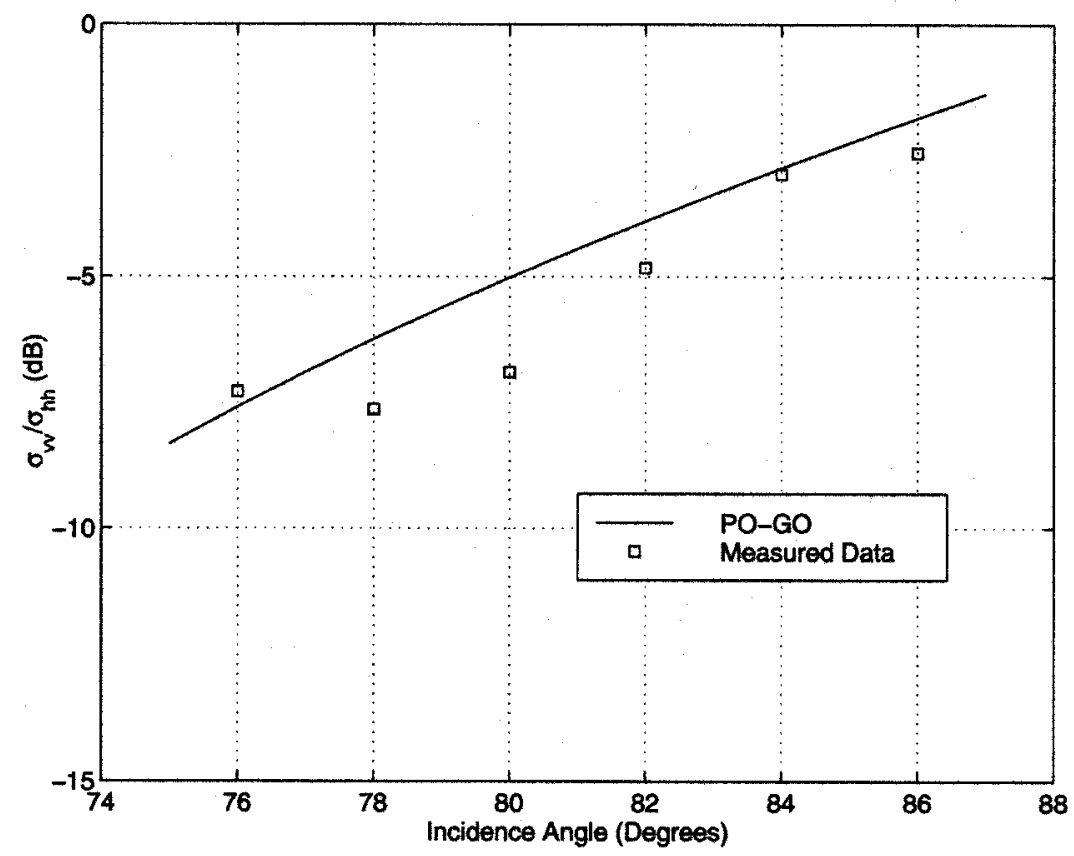

Fig. 4. The comparison between the simulation results and the measured data for the backscatter RCS ratio $\sigma_{v v} / \sigma_{h \ell}$ of the brick on asphalt surfaces at an azimuthal angle of $0^{\circ}$.

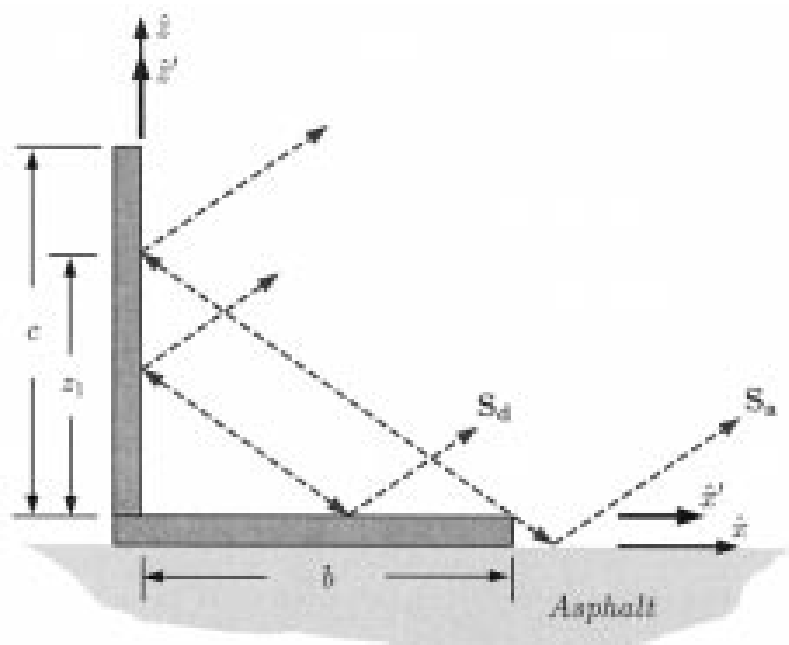

Fig. 5. The scattering mechanisms of the backscatter response of the right-angled iron on asphalt surfaces at an azimuthal angle of $0^{\circ}$.

amplitude of a dielectric plate with arbitrary orientation is given by [7]

$$
\begin{aligned}
\mathbf{S}=\frac{j k_{\circ}}{4 \pi} \int_{\mathrm{Lit}}[ & \left(1-r^{h}\right)\left(\mathbf{E}_{\circ} \cdot \hat{t}\right)\left(\hat{n}^{\prime} \cdot \hat{k}_{i}\right) \hat{k}_{s} \\
& \times\left(\hat{k}_{s} \times \hat{t}\right)+\left(1+r^{h}\right)\left(\mathbf{E}_{\circ} \cdot \hat{t}\right) \hat{k}_{s} \\
& \times\left(\hat{n}^{\prime} \times \hat{t}\right)+Z_{\circ}\left(1-r^{v}\right)\left(\mathbf{H}_{\circ} \cdot \hat{t}\right)\left(\hat{n}^{\prime} \cdot \hat{k}_{i}\right) \hat{k}_{s} \\
& \times \hat{t}-Z_{\circ}\left(1+r^{v}\right)\left(\mathbf{H}_{\circ} \cdot \hat{t}\right) \hat{k}_{s} \\
& \left.\times \hat{k}_{s} \times\left(\hat{n}^{\prime} \times \hat{t}\right)\right] e^{j k_{\circ}\left(\hat{k}_{i}-\hat{k}_{s}\right) \cdot r^{\prime}} d s^{\prime}
\end{aligned}
$$

where $\mathbf{E}_{\circ}$ and $\mathbf{H}_{\circ}$ are the amplitudes of the incident electric and magnetic fields, $r^{v}$ and $r^{h}$ are the local Fresnel reflection coefficients of the dielectric plate, $\hat{k}_{i}=k_{x}^{i} \hat{x}+k_{y}^{i} \hat{y}+k_{z}^{i} \hat{z}$ and $\hat{k}_{s}=k_{x}^{s} \hat{x}+k_{y}^{s} \hat{y}+k_{z}^{s} \hat{z}$ are the unit vectors along the incident and scattered directions, respectively, $\hat{t}$ is the unit vector perpendicular to the local plane of incidence and is defined as

$$
\hat{t}=\frac{\hat{n}^{\prime} \times \hat{k}_{i}}{\left|\hat{n}^{\prime} \times \hat{k}_{i}\right|} .
$$

An analytical expression for $\mathbf{S}$ can be obtained based on the stationary phase approximation in which the nonexponential portion of the integrand is evaluated at the SP point. The elements of the scattering matrix are found to be

$$
\begin{aligned}
S_{v v}= & -\left[r^{v}\left(\hat{n}^{\prime} \cdot \hat{v}_{i}\right)\left(\hat{n}^{\prime} \cdot \hat{v}_{s}\right)+r^{h}\left(\hat{n}^{\prime} \cdot \hat{h}_{i}\right)\left(\hat{n}^{\prime} \cdot \hat{h}_{s}\right)\right] \\
& \times \frac{\hat{n}^{\prime} \cdot \hat{k}_{i}}{\left|\hat{n}^{\prime} \times \hat{k}_{i}\right|^{2}} Q \\
S_{v h}= & -\left[r^{v}\left(\hat{n}^{\prime} \cdot \hat{h}_{i}\right)\left(\hat{n}^{\prime} \cdot \hat{v}_{s}\right)-r^{h}\left(\hat{n}^{\prime} \cdot \hat{v}_{i}\right)\left(\hat{n}^{\prime} \cdot \hat{h}_{s}\right)\right] \\
& \times \frac{\hat{n}^{\prime} \cdot \hat{k}_{i}}{\left|\hat{n}^{\prime} \times \hat{k}_{i}\right|^{2}} Q \\
S_{h v}= & -\left[r^{v}\left(\hat{n}^{\prime} \cdot \hat{v}_{i}\right)\left(\hat{n}^{\prime} \cdot \hat{h}_{s}\right)-r^{h}\left(\hat{n}^{\prime} \cdot \hat{h}_{i}\right)\left(\hat{n}^{\prime} \cdot \hat{v}_{s}\right)\right] \\
& \times \frac{\hat{n}^{\prime} \cdot \hat{k}_{i}}{\left|\hat{n}^{\prime} \times \hat{k}_{i}\right|^{2}} Q \\
S_{h h}= & -\left[r^{v}\left(\hat{n}^{\prime} \cdot \hat{h}_{i}\right)\left(\hat{n}^{\prime} \cdot \hat{h}_{s}\right)+r^{h}\left(\hat{n}^{\prime} \cdot \hat{v}_{i}\right)\left(\hat{n}^{\prime} \cdot \hat{v}_{s}\right)\right] \\
& \times \frac{\hat{n}^{\prime} \cdot \hat{k}_{i}}{\left|\hat{n}^{\prime} \times \hat{k}_{i}\right|^{2}} Q
\end{aligned}
$$

with

$$
Q=-\frac{j k_{\circ}}{4 \pi} \int_{\text {Lit }} e^{j k_{\circ}\left(\hat{k}_{i}-\hat{k}_{s}\right) \cdot \mathbf{r}^{\prime}} d s^{\prime}
$$




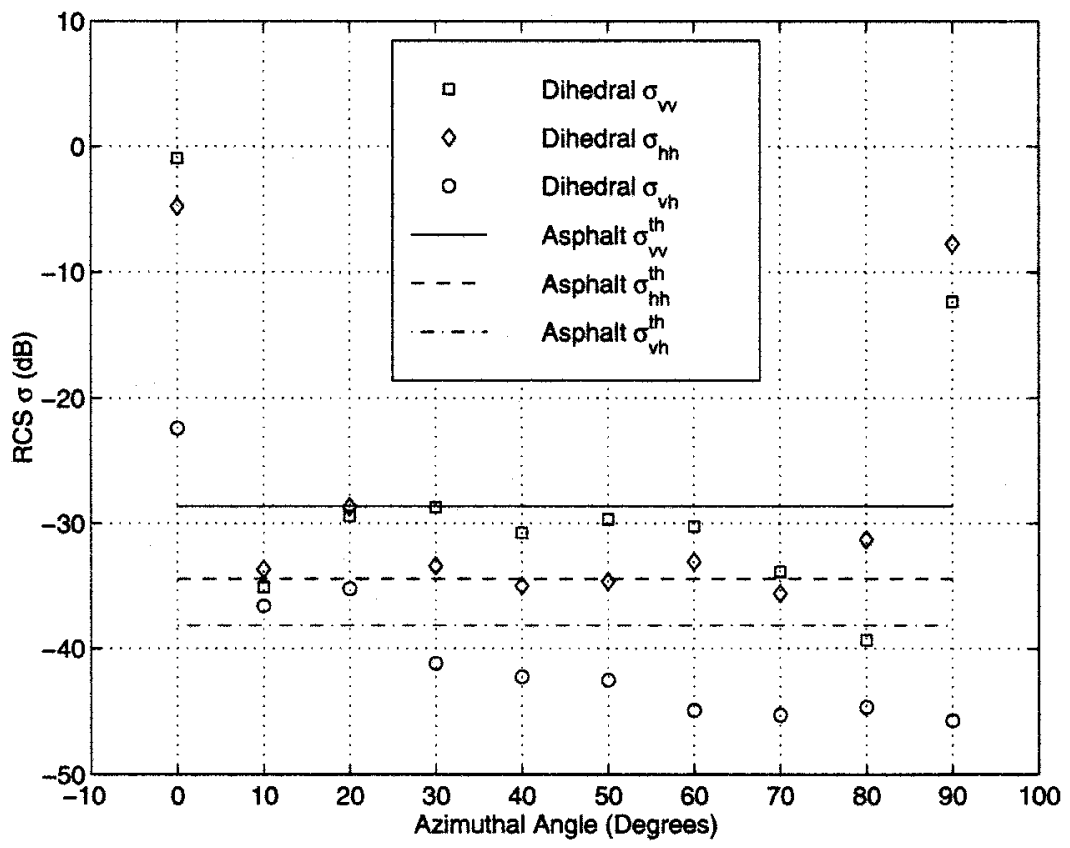

Fig. 6. The backscatter response of the dihedral on asphalt surfaces at an incidence angle of $80^{\circ}$.

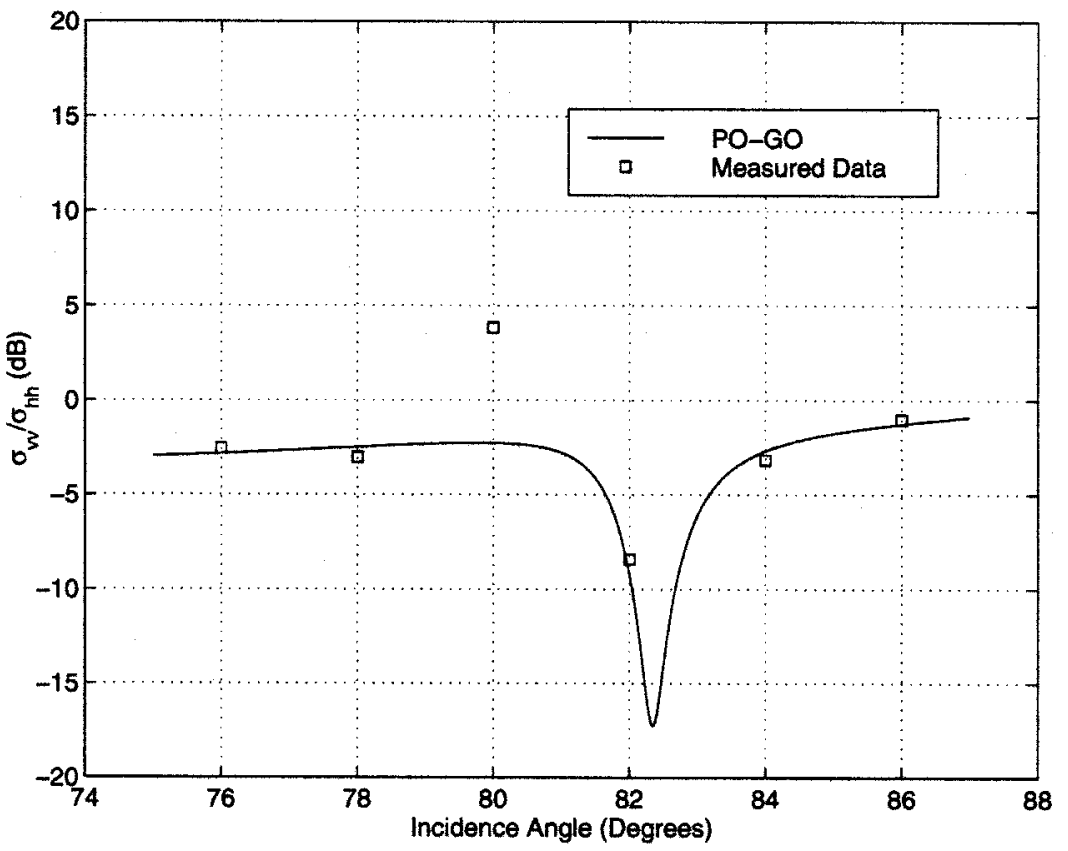

Fig. 7. The comparison between the simulation results and the measured data for the backscatter RCS ratio $\sigma_{v v} / \sigma_{h t}$ of the dihedral on asphalt surfaces at near $0^{\circ}$ azimuthal angle.

To compute the backscatter from this plate when it is placed above a dielectric ground plane, the bistatic direction $\left(\hat{k}_{s}\right)$ must be chosen so that the reflected $\hat{k}_{s}$ from the asphalt surface is along $-\hat{k}_{i}$ direction. The unit vector in the backscatter direction $\hat{k}_{r}$ is related to $\hat{k}_{s}$ by

$$
\hat{k}_{r}=\hat{k}_{s}-\left(2 \hat{z}^{\prime} \cdot \hat{k}_{s}\right) \hat{z}^{\prime}=-\hat{k}_{i} \text {. }
$$

Hence, $k_{x}^{s}=-k_{x}^{i}, k_{y}^{s}=-k_{y}^{i}$, and $k_{z}^{s}=k_{z}^{i}$. For a brick with vertical facets, the evaluation of the integral given by (7) is rather simple. Referring to Fig. 1 and recognizing significant backscatter originates only from two brick surfaces, namely side $b 1$ and side $b 2$, the expressions of $Q$ for both sides have the following form:

$$
\begin{aligned}
Q_{b 1} & =-\frac{j k_{\mathrm{o}} A_{b 1}}{2 \pi} \frac{\sin U}{U} \\
Q_{b 2} & =-\frac{j k_{\circ} A_{b 2}}{2 \pi} e^{-j U} e^{j V} \frac{\sin V}{V}
\end{aligned}
$$

where $A_{b 1}$ and $A_{b 2}$ are the areas of $b 1$ and $b 2$ sides, respectively. $U$ and $V$ are given by

$$
U=k_{\circ} k_{x}^{i} a \quad V=k_{\circ} k_{y}^{i} b,
$$




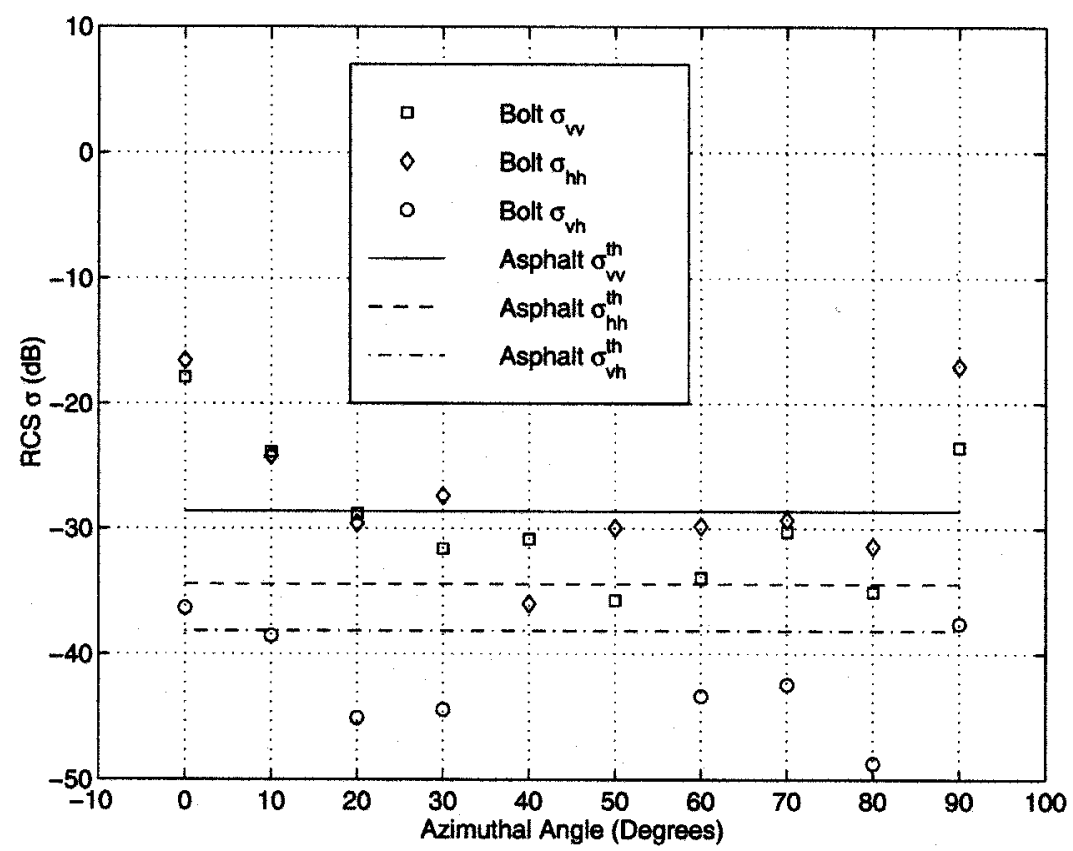

Fig. 8. The backscatter response of the bolt on asphalt surfaces at an incidence angle of $80^{\circ}$.

and $a$ and $b$ are the dimensions of the brick. The total backscatter from a plate above a ground plane is composed of two components: 1) the bistatic scattering from the plate reflected off of the ground plane, and 2) bistatic scattering from the plate when illuminated by the reflected incident wave (illumination by the image wave). The scattering matrix associated with the first term is denoted by $S_{b a}$ and is given by

$$
S_{b a}=\left[\begin{array}{cc}
r_{a}^{v} & 0 \\
0 & r_{a}^{h}
\end{array}\right]\left[\begin{array}{cc}
S_{v v, b} & S_{v h, b} \\
S_{h v, b} & S_{h h, b}
\end{array}\right]
$$

where $r_{a}^{v}$ and $r_{a}^{h}$ are the local Fresnel reflection coefficients for the asphalt surface. The scattering matrix associated with the second term denoted by $\boldsymbol{S}_{a b}$ can be obtained by taking the transpose of $\boldsymbol{S}_{b a}$ according to the reciprocity theorem. The total scattering matrix $\boldsymbol{S}_{\text {brick }}$ is the coherent sum of $\boldsymbol{S}_{a b}$ and $\boldsymbol{S}_{b a}$

$$
S_{\mathrm{brick}}=S_{a b}+S_{b a}=\left(S_{b a}\right)^{T}+S_{b a}
$$

To examine the validity of this formulation, a brick of size $20 \mathrm{~cm} \times 10 \mathrm{~cm} \times 5.7 \mathrm{~cm}$ is placed on top of the finite asphalt surface for backscatter measurement. The orientation of the brick with the $20 \mathrm{~cm} \times 5.7 \mathrm{~cm}$ side facing the radar system is defined as the $0^{\circ}$ azimuthal angle. At incidence angles from $76^{\circ}$ to $86^{\circ}$, the backscatter response of the brick was measured as a function of azimuthal angle. Five independent samples were collected for each angle. Fig. 2 shows the backscatter response of the brick on the asphalt surface as a function of azimuthal angle at an incidence angle of $80^{\circ}$. The threshold values defined for MFAR of $10^{-5}$ are included to determine the detection of the brick on asphalt surfaces $\left(3 \mathrm{~dB}\right.$ footprint area $\simeq 0.021 \mathrm{~m}^{2}$ ). Excellent detectability is observed at $0^{\circ}$ and $90^{\circ}$ azimuthal angles. It is also noticed that the $h h$ polarization offers the best separation between the brick and the asphalt response. Therefore, it is the preferred polarization to detect bricks on asphalt surfaces. To compare the analytical solution with the measured backscatter response of the brick on the asphalt surface, the effective dielectric constant of the brick is needed. An L-band microstrip ring resonator [8] may be used to estimate the effective dielectric constant of bricks at $94 \mathrm{GHz}$ since the real part of the effective dielectric constant is almost independent of frequency. The effective dielectric constant was found to be $3.67+j 0.063$. At an incidence angle of $80^{\circ}$, the simulation results based on the P. O. model for the backscatter RCSs of the brick on asphalt surfaces are compared with the measured data at azimuthal angles near $0^{\circ}$ and $90^{\circ}$, as shown in Fig. 3. In this comparison, $\pm 0.5^{\circ}$ uncertainty is allowed in the azimuthal misalignment of the brick with respect to the radar boresight. These errors can be attributed to the mechanical tolerance of the turntable used for these measurements. Fig. 4 shows the comparison between the theoretical values and the measured data of the backscatter RCS ratio $\sigma_{v v} / \sigma_{h h}$ at near $0^{\circ}$ azimuthal angle, which shows a relatively good agreement considering the fact that the surface of the brick is not exactly flat.

The same PO model can be applied to right-angled iron with some minor modifications. In this case, as shown in Fig. 5, depending on the incidence angle, part of the scattered signal from the vertical plate is reflected off of the perfectly conducting surface and part of it is reflected off of the asphalt surface. Accounting for the appropriate reflection coefficients and considering the phase difference of the backscatter due to the thickness of the metal, the scattering from each part can be added coherently. A dihedral is chosen to simulate the backscatter response of scrap metal on the asphalt surface. This dihedral has a metal 

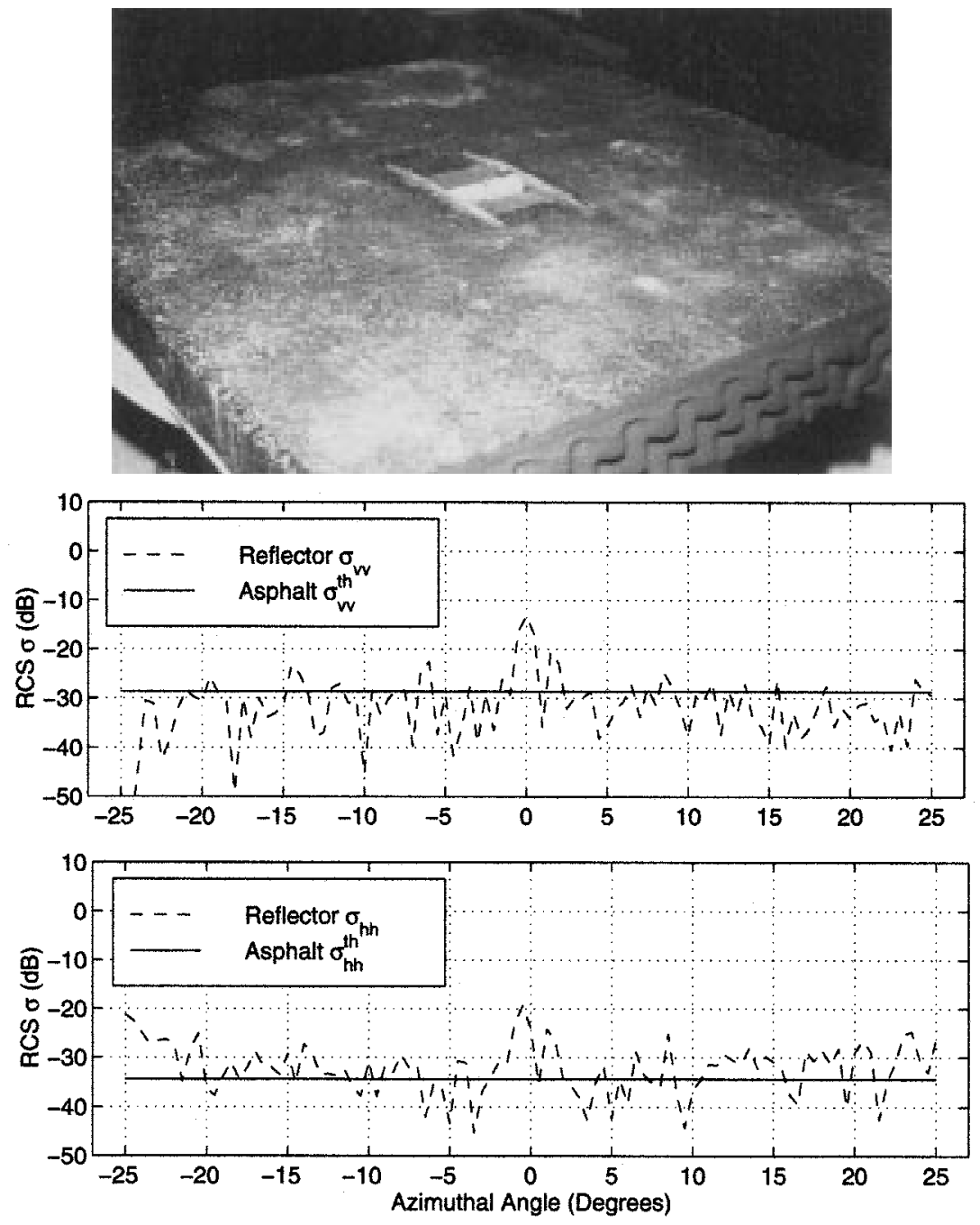

Fig. 9. The picture and the backscatter response of the reflector half-buried in asphalt mixtures at an incidence angle of $80^{\circ}$.

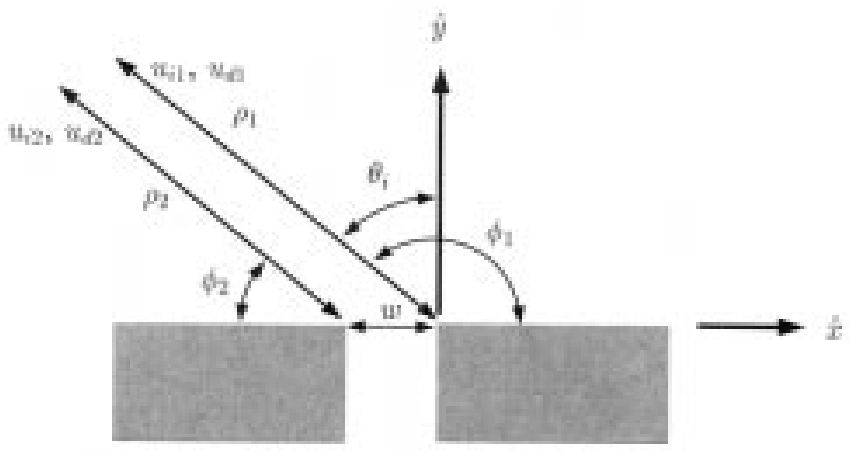

Fig. 10. The crack modeled by two right-angled impedance wedges next to each other.

thickness of $0.6 \mathrm{~cm}$ and inner dimension of $4.5 \mathrm{~cm} \times 4.5 \mathrm{~cm}$ $\times 5 \mathrm{~cm}$. The $0^{\circ}$ azimuthal angle is defined as the orientation with the open angle of the dihedral facing the radar system. The backscatter measurement of the dihedral on the asphalt surface was conducted at the same incidence angles as for the brick. The results at an incidence angle of $80^{\circ}$ are shown in Fig. 6 . A backscatter pattern similar to that of the brick is observed.

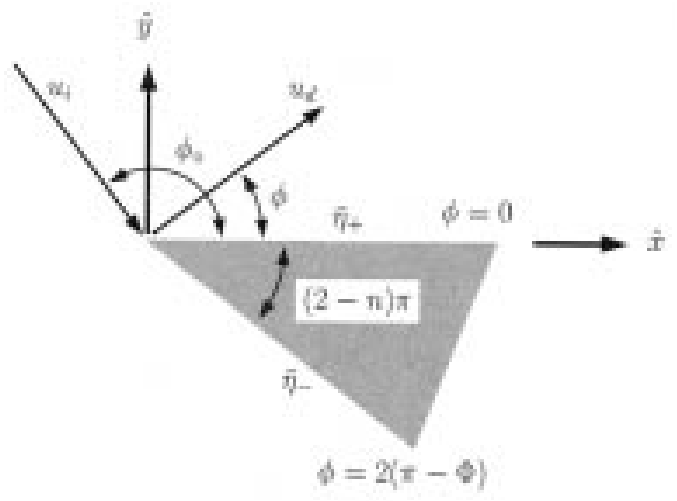

Fig. 11. The wedge geometry and the coordinate system.

Good detection can be obtained at azimuthal angles of $0^{\circ}$ and $90^{\circ}$. The $h h$ polarization is the preferred polarization for dihedral detection on asphalt surfaces. The simulation results based on the P. O. model for the backscatter RCS ratio $\sigma_{v v} / \sigma_{h h}$ at near $0^{\circ}$ azimuthal angle are compared with the experimental results. Fig. 7 shows excellent consistency between the theoretical values and the measured data. 


\section{B. Other Point Targets}

The backscatter of a bolt and an embedded lane-guide reflector are presented as other typical objects on asphalt surfaces. The bolt investigated has a length of $7.5 \mathrm{~cm}$ and a $3-\mathrm{cm}$ head. The $0^{\circ}$ azimuthal angle is defined as that orientation with the bolt head pointing toward the radar system. Fig. 8 shows the backscatter response of the bolt at an incidence angle of $80^{\circ}$. The threshold values are also included for comparison. Similar conclusions are drawn as for the brick and the dihedral.

Another type of target frequently encountered in the highway environment are structures for lane-guiding purpose. Examples are cylindrical poles, yellow paint, and reflectors. The reflectors are chosen for this study because of their strong backscatter response and widespread usage. Most reflectors are made of iron with a plastic lens in the central area. The iron is half-buried in the asphalt and the lens is tilted for light reflection, as shown in Fig. 9(a). The $0^{\circ}$ azimuthal angle is defined by the orientation for which the normal direction of the lens points at the radar system. The backscatter response of a reflector at an incidence angle of $80^{\circ}$ is shown in Fig. 9(b). It is observed that the maximum backscatter response occurs at a $0^{\circ}$ azimuthal angle. The $h h$ polarization is again the preferred choice for reflector detection.

\section{ROAD-SURFACE FAULTS}

In this section, the polarimetric backscatter behavior of typical road-surface faults is considered. Two types of faults are examined: 1) road-surface cracks, and 2) potholes. Although cracks do not pose any safety threat, the study of their backscatter response is important as far as the false-alarm rate caused by cracks is concerned. On the other hand, detection of potholes is important as they degrade the driving conditions and can lead to vehicle damage or even cause fatal accidents.

The cracks seen on road surfaces are oriented in various directions. Only those cracks oriented perpendicular to antenna boresight have significant backscatter response and will be characterized here. Two finite asphalt surfaces were placed together with a gap left in between to simulate a crack for indoor measurement. At incidence angles of $76^{\circ}, 80^{\circ}$, and $84^{\circ}$, the backscatter measurements were conducted for cracks of widths $2.54 \mathrm{~cm}$ and $5.08 \mathrm{~cm}$. For the footprint size used in this experiment, the volume-scattering contribution from the asphalt mixtures in comparison to the backscatter from the cracks is insignificant and can be ignored. At least 10 independent samples were used in the crack measurements. The measured backscatter response of cracks is reported in terms of the average backscatter power per unit length $(\mathrm{dBsm} / \mathrm{m})$, dividing the measured average RCS by the effective illuminated length.

To predict the backscatter response of cracks a first-order diffraction solution of two adjacent impedance wedges are considered (see Fig. 10). Fig. 11 shows the wedge geometry and the coordinate system. For an impedance wedge illuminated by a plane wave

$$
\mathbf{u}^{i}\left(\mathbf{E}^{i} \text { or } \mathbf{H}^{i}\right)=e^{-j k_{0} \rho \cos \left(\phi-\phi_{\circ}\right)} \hat{z}
$$

at normal incidence, the diffracted field can be expressed in terms of the nonuniform diffraction coefficient $D^{n u}$ as [9], [10]

$$
u_{z}^{d}=\frac{e^{j k_{0} \rho}}{\sqrt{\rho}} D^{n u}\left(\phi, \phi_{\circ}, \theta_{+}, \theta_{-}\right)
$$

where $\phi$ and $\phi_{0}$ are the observation and incidence angles, $\theta_{+}$ and $\theta_{-}$are the angles which satisfy the following relationship:

$$
\sin \left(\theta_{ \pm}\right)= \begin{cases}\bar{\eta}_{ \pm}, & (v \text { polarization }) \\ 1 / \bar{\eta}_{ \pm}, & (h \text { polarization })\end{cases}
$$

$\bar{\eta}_{ \pm}$are the normalized impedances of the two faces of the wedge. The nonuniform diffraction coefficient $D^{n u}$ has the following expression:

$$
\begin{gathered}
D^{n u}\left(\phi, \phi_{\circ}, \theta_{+}, \theta_{-}\right) \\
=\frac{-j e^{-j(\pi / 4)}}{n \sqrt{2 \pi k_{0}}} \frac{\sin \left(\frac{\phi_{0}}{n}\right)}{\Psi\left(\frac{n \pi}{2}-\phi_{0}\right)}\left[\frac{\Psi\left(\pi+\frac{n \pi}{2}-\phi\right)}{\cos \left(\frac{\pi-\phi}{n}\right)-\cos \left(\frac{\phi_{0}}{n}\right)}\right. \\
\left.-\frac{\Psi\left(-\pi+\frac{n \pi}{2}-\phi\right)}{\cos \left(\frac{\pi+\phi}{n}\right)-\cos \left(\frac{\phi_{\circ}}{n}\right)}\right]
\end{gathered}
$$

where $\Psi(\alpha)$ is the Maliuzhinets meromorphic function defined as

$$
\begin{aligned}
\Psi(\alpha)= & \Psi_{\Phi}\left(\alpha+\Phi+\frac{\pi}{2}-\theta_{+}\right) \Psi_{\Phi}\left(\alpha-\Phi-\frac{\pi}{2}+\theta_{-}\right) \\
& \times \Psi_{\Phi}\left(\alpha+\Phi-\frac{\pi}{2}+\theta_{+}\right) \Psi_{\Phi}\left(\alpha-\Phi+\frac{\pi}{2}-\theta_{-}\right) .
\end{aligned}
$$

The approximate formulation for the Maliuzhinets function $\Psi_{\Phi}(z)$ depends on the amplitude of the argument. The expressions are available in the literature by Senior and Herman [9], [10]. A crack can be considered to be two right-angled impedance wedges next to each other, as shown in Fig. 10. For right-angled impedance wedges, the parameters $n$ and $\Phi$ have values of 1.5 and $0.75 \pi$, respectively. The two-dimensional (2-D) RCS, $\sigma_{2 \mathrm{D}}$, is defined as

$$
\sigma_{2 \mathrm{D}}=\lim _{\rho \rightarrow \infty} 2 \pi \rho \frac{\left|u^{d}\right|^{2}}{\left|u^{i}\right|^{2}}=2 \pi\left|D^{n u}\left(\phi, \phi_{0}, \theta_{+}, \theta_{-}\right)\right|^{2} .
$$

The RCS of the crack $\sigma_{2 \mathrm{D}}^{c}$ can be obtained by adding the diffracted fields from two wedges coherently, then applying (15) to give the expression

$$
\begin{aligned}
\sigma_{2 \mathrm{D}}^{c}=2 \pi \mid & D_{1}^{n u}\left(\phi_{1}, \phi_{\mathrm{O} 1}, \theta_{+}, \theta_{-}\right) \\
& +\left.e^{-2 j k_{0} \sin \theta_{i} w} D_{2}^{n u}\left(\phi_{2}, \phi_{\circ 2}, \theta_{+}, \theta_{-}\right)\right|^{2}
\end{aligned}
$$

The measured quantity is the 3-D RCS, $\sigma_{3 \mathrm{D}}^{c}$. Conversion from $\sigma_{2 \mathrm{D}}$ to $\sigma_{3 \mathrm{D}}$ can be accomplished by the expression given in [7]

$$
\sigma_{3 \mathrm{D}}=\frac{2 L^{2}}{\lambda} \sigma_{2 \mathrm{D}}
$$




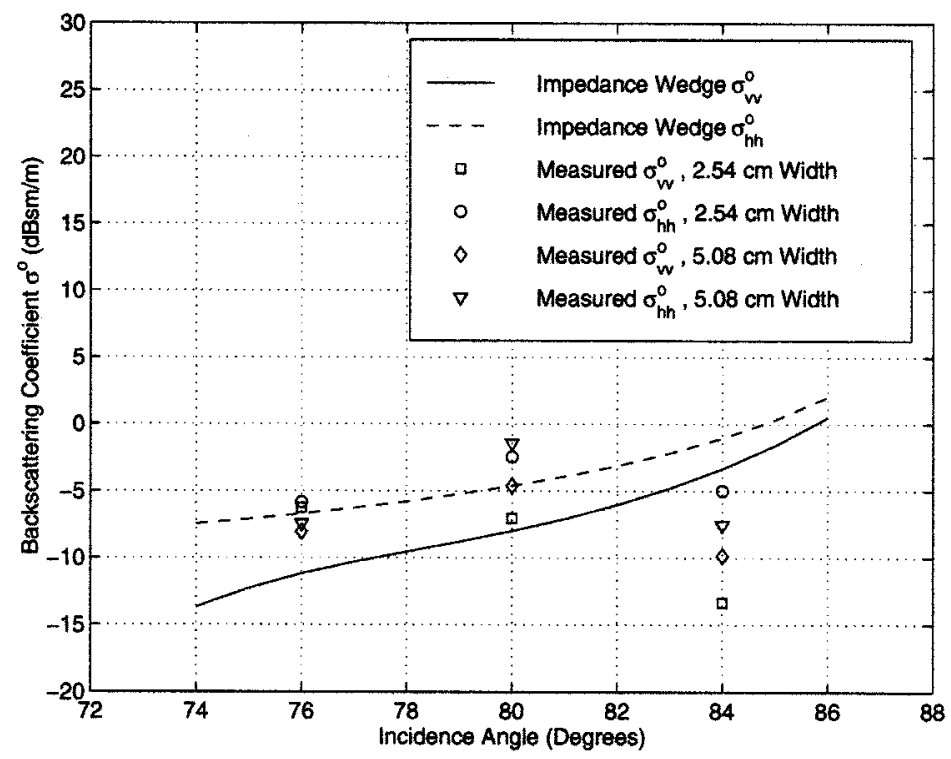

Fig. 12. The comparison between the measured results of the cracks of two widths and the simulation results based on diffraction from two right-angled impedance wedges.

TABLE I

Particle Size Distributions of Asphalt Mixtures and the Pebbles

\begin{tabular}{c|r||c|r}
\hline \multicolumn{2}{c||}{ Asphalt Mixtures } & \multicolumn{2}{c}{ Pebbles } \\
\hline \hline Sieve Size in cm & \% Passing & Sieve Size in cm & \% Passing \\
\hline 1.27 & 100 & 3.18 & 100 \\
\hline 0.95 & 92 & 2.54 & 97.8 \\
\hline 0.64 & 75 & 2.22 & 95.9 \\
\hline 0.32 & 62 & 1.91 & 93.2 \\
\hline 0.25 & 55 & 1.59 & 87.7 \\
\hline 0.16 & 44 & 1.27 & 77.9 \\
\hline 0.06 & 20 & 0.95 & 61.9 \\
\hline 0.03 & 7 & 0.64 & 33.3 \\
\hline 0.01 & 4.5 & \multicolumn{2}{c}{} \\
\hline
\end{tabular}

By setting $L=1 \mathrm{~m}$, the RCS per unit length of cracks is obtained.

Fig. 12 shows the comparison between the measured data and the simulation results based on diffraction from two impedance wedges. Good agreement is observed. In particular, both results indicate that the $h h$ response is greater than the $v v$ response. The slight discrepancy at lower grazing angles can be attributed to the nonideal modeling of the crack edge by a right-angled wedge at millimeter-wave frequency because of random distribution of rocks and sand along the crack edge. The results also indicate that the diffracted fields from a crack at incidence angles from $74^{\circ}$ to $86^{\circ}$ are insensitive to the crack width. This is due to the fact that the diffracted field from the impedance wedge near the source is negligible $\left(D_{1}^{n u} \gg D_{2}^{n u}\right)$.

The aforementioned disagreement at lower grazing angles can be improved by modeling the crack edge as an impedance cylinder. The radius of the cylinder is selected based on the rock size distribution of asphalt mixtures as shown in Table I. The cylinder models the curve describing the crack edge, improving the backscatter prediction. In the wedge model, it is found that the backscatter response of the crack is dominated by the diffraction from the wedge further away from the source. The same conclusion can be reached when the corners are modeled by curved surfaces. In this case a specular point on the surface of the cylinder further away from the source predominantly contributes to the backscatters. Therefore, only the backscatter response of a single impedance cylinder, representing the further crack edge, is considered here. The ensemble average of the backscatter power is calculated using the backscatter of impedance cylinders of various radii and unit length, which are chosen based on the particle size distribution. The simulation results based on the scattering from an impedance cylinder are compared with the measured data as shown in Fig. 13.

\section{A. Potholes}

The potholes seen on the road surfaces vary in shape and size. General descriptions of the backscatter response of potholes are difficult. An asphalt slab with a cylindrical hole of $10 \mathrm{~cm}$ diameter is used to simulate the pothole as shown in Fig. 14(a). The backscatter response of the pothole was measured at incidence angles from $74^{\circ}$ to $86^{\circ}$. A pothole of depth $3.81 \mathrm{~cm}$ was considered. Five independent samples were collected for each incidence angle. The results are shown in Fig. 14(b) along with the comparison with the threshold values. It is observed that the $h h$ polarization is the preferred polarization for pothole detection.

\section{ROADSIDE BOUNDARIES}

The backscatter behavior of roadside boundaries is of interest because of their application in autonomous vehicle control. The backscatter response of roadside boundaries is studied in this section. Typical roadside boundaries are curbs, guardrails, and gravel road shoulders. Backscatter measurements of roadside boundaries were conducted outdoors. Most of the roadside boundaries examined here are considered as 1-D distributed targets. Therefore, the ensemble backscatter power per unit 


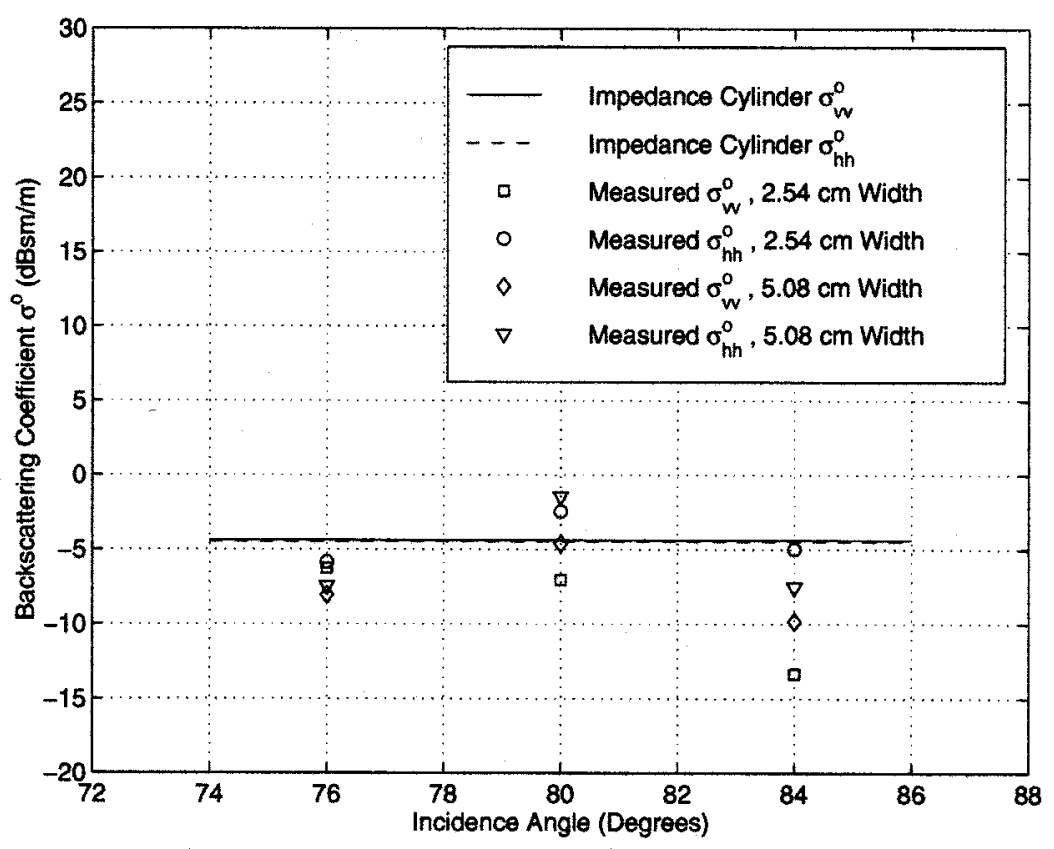

Fig. 13. The comparison between the measured results of the cracks of two widths and the simulation results based on scattering from an impedance cylinder.
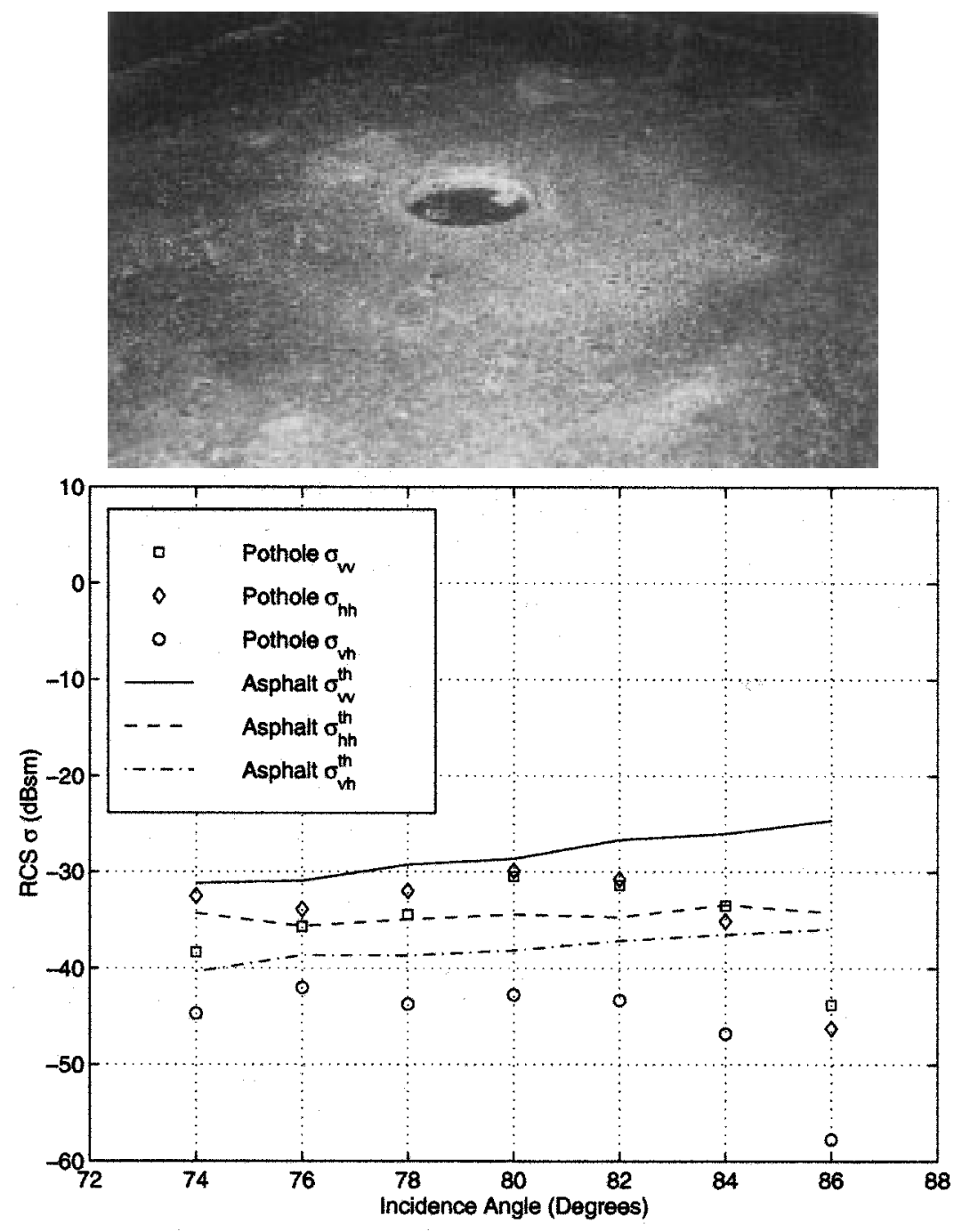

Fig. 14. The picture and the backscatter response of the pothole of depth $3.81 \mathrm{~cm}$. 


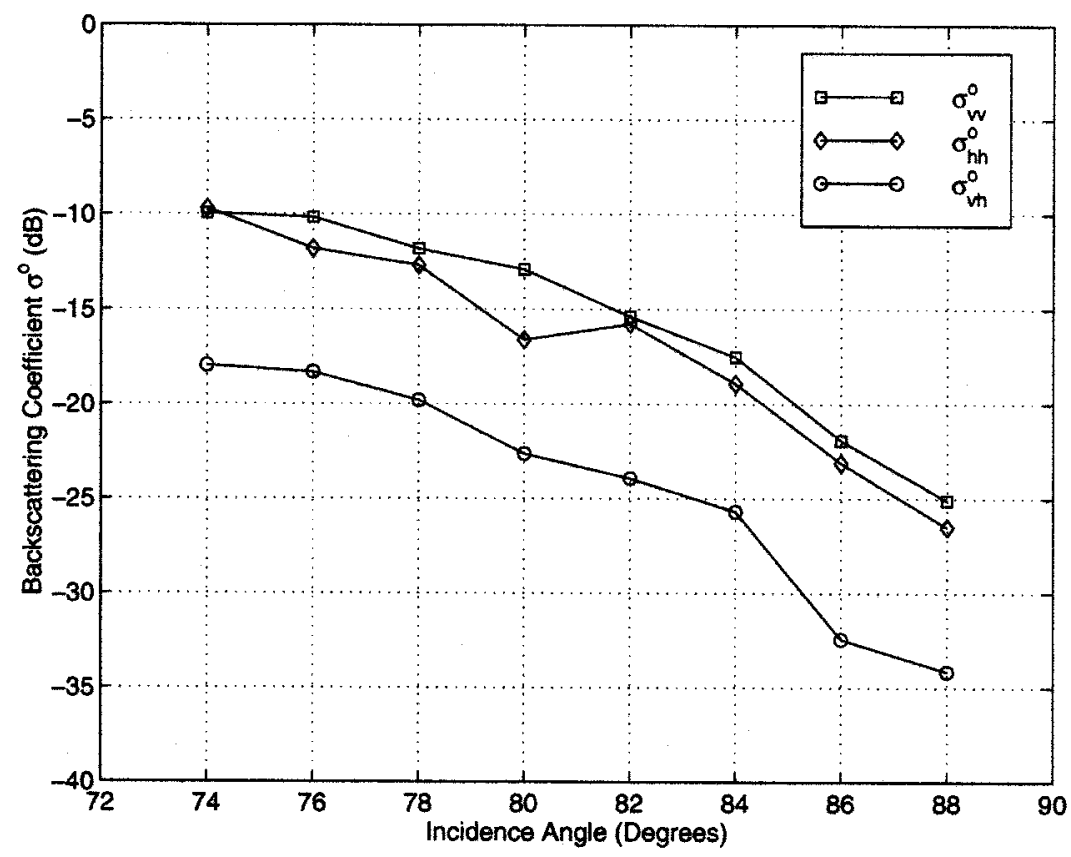

Fig. 15. The backscatter response of the pebbles.

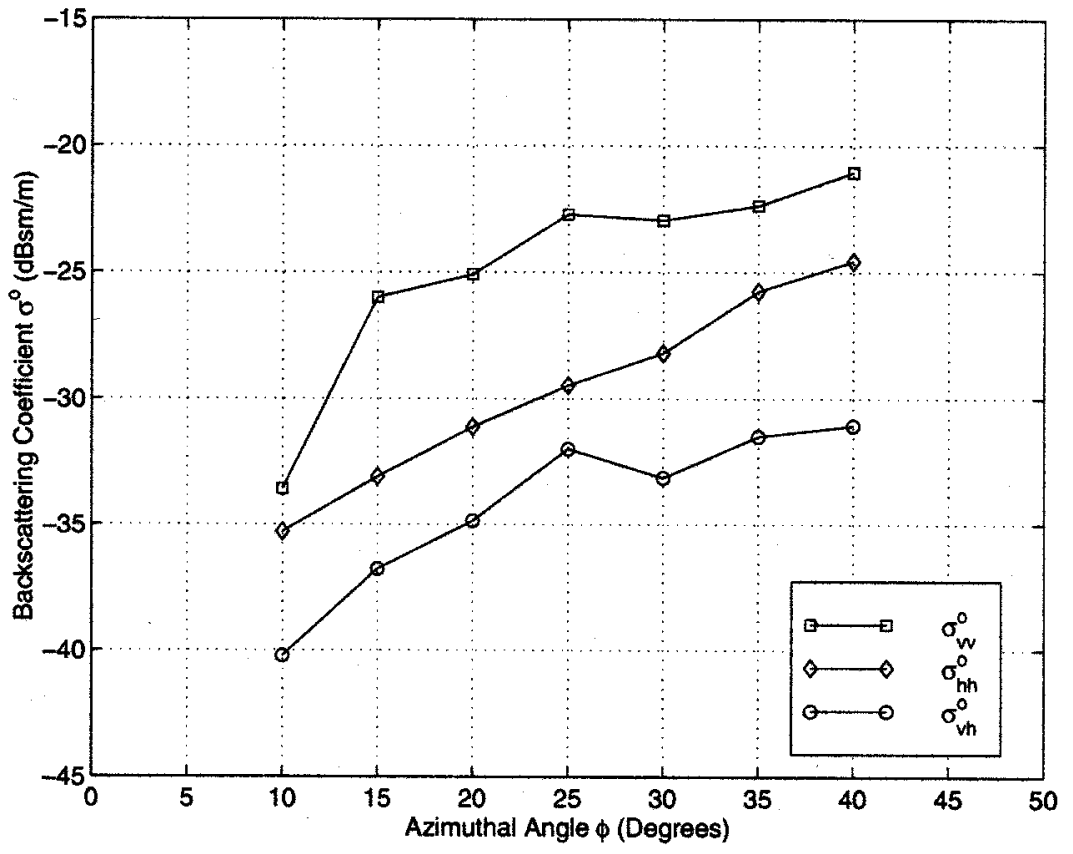

Fig. 16. The polarimetric backscatter response of a concrete curb.

length (rather than the RCS) is reported. Their backscatter response was measured at one specific incidence angle and expressed as a function of azimuthal angle.

Most commonly, gravels and pebbles are used as road shoulders. The selection of rocks for roadside depends on availability. The backscatter response of a surface covered by pebbles is determined by the dielectric properties of the rocks, size distribution, and surface roughness statistics. The size distribution of the pebbles in this investigation is listed in Table I. The backscatter measurements were conducted at incidence angles in the range of $74^{\circ}-88^{\circ}$. Eighty independent samples were collected for each incidence angle. The backscatter response of the pebbles is shown in Fig. 15.

\section{A. Curbs}

The curb is considered to be a 1-D distributed target. The transverse dimension of the curb examined here has a vertical height of $14 \mathrm{~cm}$, upper horizontal length of $15.3 \mathrm{~cm}$ (next to 


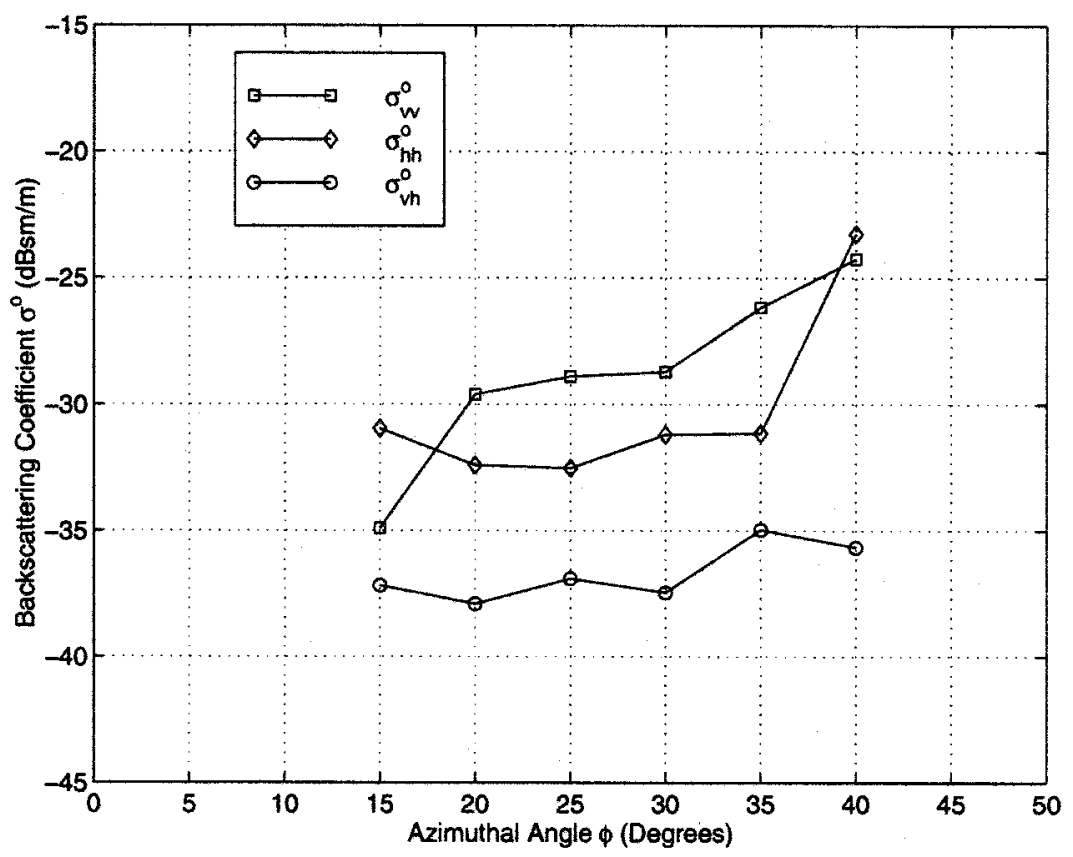

Fig. 17. The polarimetric backscatter response of a metallic guardrail.

the grass), and lower horizontal length of $38 \mathrm{~cm}$ (next to the asphalt). The backscatter measurements were conducted at an incidence angle of $82^{\circ}$; thirty independent samples were taken. Fig. 16 shows the backscatter response as a function of azimuthal angle $\phi$, where $\phi$ is defined as the angle between the antenna boresight and the curb.

\section{B. Guardrails}

Guardrails vary in height, size, and the distance between poles. The guardrail in this investigation has a height of $50 \mathrm{~cm}$ (from the center to the ground), width of $33 \mathrm{~cm}$, and a distance between poles of $2.2 \mathrm{~m}$. The backscatter measurements were conducted at an incidence angle of $84^{\circ}$. Thirty independent samples were taken and the ensemble backscatter power per unit length is shown in Fig. 17.

\section{CONCLUSION}

In this paper, the near-grazing backscatter behavior of debris on road surface and surface faults is investigated at W-band frequencies. For targets with planar facets, a physical optics scattering model is developed. This model is tested against the backscatter measurements of a brick and right-angled iron on an asphalt surface. The backscattering coefficients of asphalt surfaces are used to determine a threshold value for the detectability of the point targets based on a specified false alarm rate. Also two theoretical models, one based on diffraction from impedance wedges and the other one based on scattering from impedance cylinders, are used to predict the backscatter response of a crack. It is shown that both these models are capable of predicting the backscatter response from a crack reasonably. The polarimetric measurements of various roadside boundaries are also included. The experimental results indicate that the detectability of targets on the road and surface faults is a function of the azimuthal orientation of the targets as well as the system parameters such as incidence angle and polarization. Since the backscatter from asphalt surfaces assumes its lowest value at $h h$, polarization whereas most targets show a maximum RCS at $h h$ polarization, $h h$ polarization was found to be the most suitable polarization for target detection.

\section{REFERENCES}

[1] K. Sarabandi, E. S. Li, and A. Nashashibi, "Modeling and measurements of scattering from road surfaces at millimeter-wave frequencies," IEEE Trans. Antennas Propagat., vol. 45, Nov. 1997.

[2] E. S. Li and K. Sarabandi, "Low grazing incidence millimeter-wave scattering models and measurements for various road surfaces," , Apr. 1998, submitted for publication.

[3] K. Sarabandi and E. S. Li, "Characterization of optimum polarization for multiple target discrimination using genetic algorithms," IEEE Trans. Antennas Propagat., vol. 45, pp. 1810-1817, Dec. 1997.

[4] G. D. Maliuzhinets, "Some generations of the method of reflections in the theory of sinusoidal wave diffraction," Ph.D. dissertation (in Russian), Fiz. Inst. Lebedev, Acad. Nauk. U.S.S.R..

[5] - "Excitation, reflection and emission of surface waves from a wedge with given face impedances," Sov. Phys. Doklady, vol. 3, pp. 752-755, 1958 .

[6] A. Nashashibi, K. Sarabandi, and F. T. Ulaby, "A calibration technique for polarimetric coherent-on-receive radar system," IEEE Trans. Antennas Propagat., vol. 43, pp. 396-404, Apr. 1995.

[7] K. Sarabandi, "Electromagnetic scattering from vegetation canopies," Ph.D. dissertation, The University of Michigan, 1989.

[8] K. Sarabandi and E. S. Li, "A microstrip ring resonator for soil moisture measurements," IEEE Trans. Geosci. Remote Sensing, vol. 35, Sept. 1997.

[9] T. B. A. Senior and J. L. Volakis, Approximate Boundary Conditions in Electromagnetics. London, U.K.: IEE Press, 1995, ch. 4.

[10] M. Herman, "High frequency scattering from canonical impedance structures," Ph.D. dissertation, Univ. Michigan, Ann Arbor, 1987. 


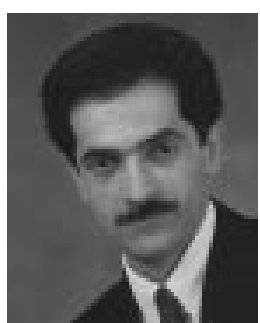

Kamal Sarabandi (S'87-M'90-SM'92-F'00) received the B.S. degree in electrical engineering from Sharif University of Technology, Tehran, Iran, in 1980. From 1980 to 1984 he worked as a Microwave Engineer in the Telecommunication Research Center. $\mathrm{He}$ entered the graduate program at the University of Michigan in 1984 and received the M.S.E. degree in electrical engineering in 1986, the M.S. degree in mathematics, and the Ph.D. degree in electrical engineering, in 1989.

He is presently the director of the Radiation Laboratory in the Department of Electrical Engineering and Computer Science at the University of Michigan. He has 18 years of experience with microwave sensors and radar systems. In the past eight years, he has served as the Principal Investigator and Co-Investigator on many projects sponsored by NASA, JPL, ARO, ONR, ARL, NSF, and numerous industries. He has published many book chapters and more than 85 papers in refereed journals on electromagnetic scattering, random media modeling, wave propagation, microwave measurement techniques, radar calibration, application of neural networks in inverse scattering problems, and microwave sensors. He has also had more than 150 papers and invited presentations in national and international conferences and symposia on similar subject.

Dr. Sarabandi is listed in American Men \& Women of Science and Who's Who in Electromagnetics. He is a Member of the IEEE Geoscience and Remote Sensing ADCOM since January of 1998 and served as the Chairman of Geoscience and Remote Sensing Society Southeastern Michigan chapter from 1992-1998. He is also a member of Commission F of URSI and of The Electromagnetic Academy. He was a Recipient of a 1996 Teaching Excellence Award, the 1997 Henry Russel Award from the Regent of The University of Michigan, and the 1999 GAAC Distinguished Lecturer Award from the German Federal Ministry for Education, Science, and Technology.

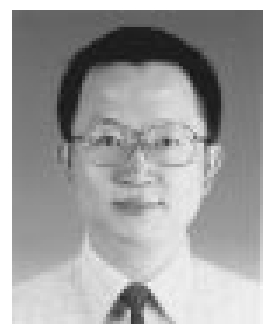

Eric S. Li received the B.S. degree from the Tamkang University, Taiwan, R.O.C., in 1986. He received the M.S. degree in electrical engineering from the State University of New York, Stony Brook, in 1987 and the $\mathrm{Ph} . \mathrm{D}$. degree in electrical engineering from the University of Michigan, Ann Arbor, in 1998.

From 1988 to 1992 and 1998 to 1999 , he was in cellular phone industries as a microwave engineer participating in the design of microwave circuits and antenna. Since August 1999, he has been with the Department of Electrical Engineering, National Chi Nan University, Taiwan, R.O.C., as an Assistant Professor. His current research interests include polarimetric millimeter-wave radar systems, calibration and measurement techniques, electromagnetic scattering and millimeter-wave remote sensing. 